\title{
FIB Dual-Beam Sample Preparation for TEM Observation
}

\author{
Geoffroy Auvert
}

STMicroelectronics, CEA-LETI-SCPC, 17 rue des martyrs, 38054 Grenoble, Cedex, France

Etching of integrated circuits using Focus ion beam (FIB) produces samples for Transmission Electron Microscopy (TEM) observation. Such a sample preparation is compatible with the objective of imaging circuits having different density area. An area composed with very heavy atoms imposes a larger diffraction of the TEM electrons and the beam intensity is locally lowered. This kind of TEM image is currently made after normal FIB sample preparation. This FIB sample preparation procedure uses an ion beam moving quickly in one direction and very slowly in the perpendicular direction. By measuring the etching rate at various ion beam current according to this procedure, it has been found that the relation between depth, speed and beam current is not simple. Fortunately, this procedure gives a sample with some usable thickness and a TEM analysis is quite possible. Most of the time, the image can be interpreted and the sample preparation is acceptable. This TEM imaging method is largely used to image transistors or connections in integrated circuits.

Unfortunately, some applications need an other kind of TEM imaging technique. This other kind of image must show something, which is not related with the atom density of each area but to an other modification of the beam intensity. For example, electrical potential imaging using TEM observation in MOS transistors is an up-to-day objective, which is not solved in a normal TEM. The objective of this application is to compare several observed area, so we need a sample thickness very homogenous. To build such a homogenous thickness, the sample preparation must be specific and very well controlled. The objective of this abstract is to describe the use of such a special FIB dual-beam sample preparation technique to solve this problem.

First of all, we have to set special FIB conditions to make a wall shaped sample. The second step describes the technique using an electron beam to perform an "in situ" wall thickness measurement.

The first step to build a perfect wall is to use a software called the "moving box" software. Description of this technique is not the object of this abstract because some other techniques do not need it completely. Whatever, after this etching, the sample looks like a wall and the main problem to solve is to measure the wall thickness.

Usually, the thickness measurement is performed by making an electron beam image of the wall. In this image, both side surfaces of the wall are visible. As a result, it is found that when the wall is thick, the apparent value of the wall thickness is nearly equal to those measured with the TEM. Unfortunately, this simple measurement does not give the real thickness value. This is due to the very high image brightness increase of the electron impinging on the side area of the wall. Therefore, we cannot determine where is the wall surface and cannot deduce the real wall thickness. In order to solve this difficulty, it should be possible to image the wall with the electron beam but without any enhancement of the secondary electron coming from the wall surface.

In the dual-beam tool, when the ion beam has finished an etching, the wall and the electron beam has an angle, which is very close to the angle between the FIB column and the electron column. In 
that case, by making the electron image of the wall surface, we can obtain its real image. The objective is now to measure the real wall thickness, which is in between 0.05 and $1 \mu \mathrm{m}$. The abstract is now going to describe how this thickness can be measured with an "in situ" electron beam.

The first step is to fix a detector, which can attract secondary electrons. Usually, the detector is near the electron column and an image of the surface is made. But, to solve our problem, we also need to collect secondary electrons coming from the other side of the wall. This is possible when the angle between the lines going from the sample to the detector and the electron beam direction is above $50^{\circ}$. If this is not the case, the secondary electron from the backside cannot be detected. Above $50^{\circ}$, the image of the upper surface wall is superimposed with the secondary electrons from the backside of the wall. Using such a detector, we can therefore obtain several images at various electron beam energy. Some of these images have collected backside electrons and some are without when the electron energy is small enough. By comparing all these images, it is easy to determine an energy beam threshold. The last step of the procedure is to deduce from this threshold value, the real wall thickness.

It is well established that electrons arriving from an electron column on a surface are stopped by the material [1,2]. These publications describe measurement using electron beam energy above 5 $\mathrm{keV}$ and demonstrated the use of an energy beam power of 1.75 to calculate the relation between the irradiation depth at energy up to $50 \mathrm{keV}$. As a consequence, these numbers can be used to solve sometimes our problem. Apparently, an extrapolation of the data of ref. [1,2] can be a solution. But for TEM sample observations using thickness from 0.05 to $0.3 \mu \mathrm{m}$, these data are not precise enough and three difficulties have to be mentioned. These difficulties linked to (i) the angle between the sample and the electron beam, (ii) the real value of the beam energy and (iii) the influence of the amorphised thickness on the wall.

The best way to avoid these difficulties is to calibrate the threshold beam energy through a sample with known thickness. For example, by imaging at various electron beam energy, a wedged shaped sample with a known angle, it is possible to obtain all the necessary data versus known thickness nearly down to zero. In our experiments on silicon single crystal, it has been found that the reference value is in good agreement with experimental result controlled after using TEM measurement. It has also been measured the influence of the amorphous layer on the thickness value for sample of thickness below $0.2 \mu \mathrm{m}$.

As a conclusion, some TEM techniques, which require very thin sample with very well known thickness, can be etched in FIB dual-beam. The FIB etching makes a wall having parallel sites and the "in situ" electron beam thickness measurements can be very precisely obtained for any wall at thickness value below $5 \mu \mathrm{m}$

[1] V.E.Cosslett and R.N.Thomas, Brit. J. Appl. Phys. 15(1964) 1283.

[2] T.E.Everhart and P. H. Hoff, J. Appl. Physics, Vol. 42, 5837, December 1971. 